\title{
MHD Natural Convection Flow of Fluid From a Vertical Flat Plate Considering Temperature Dependent Viscosity
}

\author{
S. F. Ahmmed ${ }^{1}$ and M. S. A. Sarker \\ ${ }^{1}$ Mathematics Discipline, Khulna University, Khulna-9208, Bangladesh \\ Department of Applied Mathematics, Rajshahi University, Rajshahi-6205, Bangladesh
}

Received 12 April 2010 accepted in revised form 18 August 2010

\begin{abstract}
A two-dimensional natural convection flow of a viscous incompressible and electrically conducting fluid past a vertical impermeable flat plate is considered in presence of a uniform transverse magnetic field. Here the viscosity is taken as dependent on temperature whereas the thermal conductivity is assumed constant. We also investigate the effect of magnetic field on the natural convection flow of a viscous incompressible and electrically conducting fluid. The effect of variable viscosity and magnetic field on local skin friction, the rate of heat transfer and the profiles for velocity as well as viscosity in the entire free convection regime are presented and discussed.
\end{abstract}

Keywords: Natural convection; Magnethydrodynamics (MHD); Viscosity; Prandtl number.

(C) 2010 JSR Publications. ISSN: 2070-0237 (Print); 2070-0245 (Online). All rights reserved.

DOI: $10.3329 /$ jsr.v2i3.4776 J. Sci. Res. 2 (3), 453-463 (2010)

\section{Introduction}

Studies of forced, free and mixed convection flow of a viscous incompressible fluid, in the absence of magnetic field, along a vertical surface have extensively been conducted by Sparrow and Gregg [1], Merkin [2], Loyed and Sparrow [3]. Hunt and Wilks [4] introduced a group of continuous transformations computation for the boundary layer equations between the similarity regimes for mixed convection flow. In the case of similarity regimes Hunt and Wilks [4] recognized $\zeta\left(=G r_{x} / R e_{x}^{2}\right.$, where $G r_{x}$ is the local Grashof number and $R e_{x}$ is the local Reynolds number), a governing parameter for the flow from a vertical plate. Forced convection exists as when $\zeta$ goes to zero, which occurs at the leading edge, and the free convection limit, can be reached at large values of $\zeta$.

\footnotetext{
${ }^{1}$ Corresponding author: sfahmmed@yahoo.com
} 
Perturbation solutions have been developed in both the cases, since both the forced convection and free convection limits admit similarity solution. Empirical patching of two perturbation solutions have also been carried out to provide a uniformly valid solution by Raju et al. [5] which covers the whole range of the values of $\zeta$. They obtained a finite difference solution applying an algebraic transformation $Z=1 /\left(1+\zeta^{2}\right)$. Considering the free convection as a perturbation quantity has developed many solutions. Tingwi et al. [6] have also studied the effect of forced and free convection along a vertical flat plate with uniform heat flux by considering that the buoyancy parameter $\zeta_{p}$ to be $G r_{x} / R e_{x}{ }^{5 / 2}$. The solutions were obtained for the small buoyancy parameter taking into the account of the perturbation technique.

Because of its application for MHD natural convection flow in the nuclear engineering where convection aids the cooling of reactors, the natural convection boundary layer flow of an electrically conducting fluid up a hot vertical wall in the presence of strong magnetic field has been studied by several authors, such as Sparrow and Cess [7], Reley [8] and Kuiken [9]. Simultaneous occurrence of buoyancy and magnetic field forces in the flow of an electrically conducting fluid up a hot vertical flat plate in the presence of a strong cross magnetic field was studied by Sing and Cowling [10] who had shown that regardless of strength of applied magnetic field there will always be a region in the neighborhood of the leading edge of the plate where electromagnetic forces are unimportant. Creamer and Pai [11] and Singh et al. [12] presented a similarity solution for the above problem with uniform heat flux by formulating it in terms of both a regular and inverse series expansions of characterizing coordinate that provided a link between the similarity states closed to and far from the leading edge. Hossain and Ahmed [13] studied the combined effect of the free and forced convection with uniform heat flux in the presence of strong magnetic field. An analysis was made by Rat and Chaudhary [14] for the steady twodimensional, laminar boundary layer flow of a viscous, incompressible, electrically conducting fluid near a stagnation point of stretching sheet in the presence of magnetic field. It was considered that the sheet is stretched in its own plane with velocity and temperature proportional to the distance from the stagnation point. Samad and Mohebujjaman [15] had investigated the effect of a chemical reaction of an electrically conducting viscous incompressible fluid on the flow over a linearly stretching vertical sheet in the presence of heat and mass transfer as well as a uniform magnetic field which is normal to the sheet with heat generation/absorption. MHD free convection in the present of temperature-dependent heat source in a viscous incompressible fluid confined between a long vertical wavy wall and a parallel flat wall in slip flow regime with constant heat flux at the flat wall was studied by Taneja and Jain [16]. Hossain et al [17] also investigated the MHD free convection flow along a vertical porous flat plate with a power law surface temperature in the presence of a variable transverse magnetic field employing two different methods namely (i) perturbation methods for small and large values of the scaled stream-wise transpiration velocity variable $\xi_{s}\left(=V_{0} \sqrt{ }\left(2 x / v U_{\infty}\right.\right.$, where $\mathrm{V}_{0}$ is the transpiration velocity) and (ii) the finite difference together with the Keller box method [18]. Wilks [19] recognized a parameter $\xi$, defined by $\xi=\left(\sigma H_{0}{ }^{2} / \rho_{\infty}\right)^{2} x / g \beta\left(T_{0}-T_{\infty}\right)$ 
to investigate the MHD free convection flow about a semi-infinite vertical plate in a strong cross magnetic field. The work of that follows reformulates the problem in terms of coordinates expansions with respect to a non-dimensional characteristic length which is fundamental to the problem in its reflection to the relative magnitudes of buoyancy and magnetic forces at varying locations along the plate. A step by step numerical solution has been obtained to supplement the series solutions for small and large $\xi$. The effect of variable viscosity is considered for the flow and heat transfer on MHD natural convection in micropolar fluids was studied by El-Hakiem and Abdou [20]. Studies of the effects of variable viscosity, heat and mass transfer on nonlinear MHD mixed convection flow in a porous medium over a wedge with chemical reaction and heat radiation in the presence of suction or injection was conducted by Kandasamy et al. [21].

In the above analysis, the solutions for the problem, Wilks [19] used only series solutions method for the case of constant viscosity and constant thermal conductivity. Ahmmed [22] studied the works of Wilks [19] for the three cases and obtained solutions using series solution method and finite difference method. The solutions of Ahmmed [22] showed an excellent agreement with the solution of Wilks [19]. Here we consider viscosity as variable which depends on temperature and finally we obtain ordinary differential equations by introducing appropriate coordinate transformations. We solve the ordinary differential equations by series solution method for small magnetic field parameter, asymptotic solution for large values of magnetic field parameter and finite difference method for all values of this parameter. The effect of the viscosity variation parameter and magnetic field parameter on the skin friction and rate of heat transfer are given in the tabular form. We also draw the velocity profiles, the viscosity profile for different values of the viscosity variation parameter and magnetic field parameter.

\section{The Governing Equations}

We consider the steady two dimensional laminar free convection boundary layer flow of a viscous incompressible and electrically conducting fluid with temperature dependent viscosity but constant thermal conductivity. Here we make the assumptions that (i) the plate is vertically semi-infinite and impermeable (ii) the transverse magnetic field of strength $H_{0}$ is uniformly distributed. The basic equations in the case of variable viscosity are as given below [22]:

$$
\begin{aligned}
& \frac{\partial u}{\partial x}+\frac{\partial v}{\partial y}=0 \\
& u \frac{\partial u}{\partial x}+v \frac{\partial u}{\partial y}=\frac{1}{\rho_{\infty}} \frac{\partial}{\partial y}\left(\mu \frac{\partial u}{\partial y}\right)+g \beta\left(T-T_{\infty}\right)-\frac{\sigma H_{0}{ }^{2} u}{\rho_{\infty}} \\
& u \frac{\partial T}{\partial x}+v \frac{\partial T}{\partial y}=\frac{\kappa}{\rho_{\infty} c_{p}} \frac{\partial^{2} T}{\partial y^{2}}
\end{aligned}
$$


with the boundary conditions

$$
\begin{aligned}
& u=v=0, \quad T=T_{0} \quad \text { at } y=0 \\
& u \rightarrow 0, \quad T \rightarrow T_{\infty} \quad \text { as } y \rightarrow \infty
\end{aligned}
$$

Here $u, v$ is the velocity components associated with the direction of increase of coordinates $x$ and $y$ measured along and normal to the vertical plate (see Fig. 1). $T$ is the temperature of the fluid in the boundary layer, $g$ is the acceleration due to gravity, $\beta$ is the coefficient of thermal expansion, $\kappa$ is the thermal conductivity, $\rho_{\infty}$ is the density of the fluid, $c_{p}$ is the specific heat at constant pressure and $T_{\infty}$ is the temperature of the ambient fluid $\mu$ is the viscosity of the fluid depending on temperature defined in equation (5). Since the viscosity decreases with the increase of temperature. Following Gray at al.[23], Mehta and Sood[24], Kafoussias and Williams [25] and Kafoussias et al. [26], we assume the temperature dependent viscosity $\mu$ can be of the form

$$
\frac{\mu}{\mu_{0}}=\frac{1}{1+\varepsilon \theta}
$$

where $\theta=T-T_{\infty}$

Here $\varepsilon$ is the viscosity variation parameter which is positive for heating, negative for cooling and zero for constant viscosity of the fluids, $\mu_{0}$ is the constant viscosity.

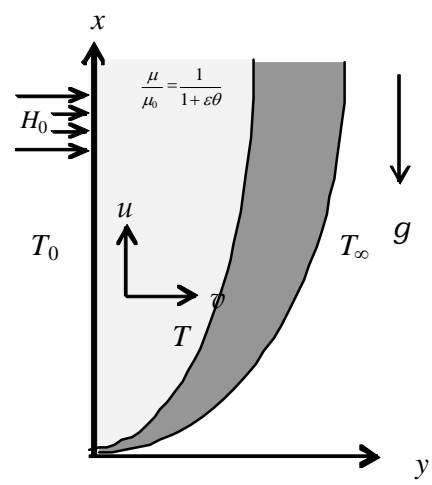

Fig. 1. The flow configuration and coordinate system.

We observe that the Eqs. (2) and (3) together with the boundary conditions (4) are non-linear partial differential equations. In the following section the solution methods for these equations are discussed details.

\section{Solution for All $\xi$}

Now we introduce the following transformations of the equation (6) to the Eqs. (2) and (3) 


$$
\begin{aligned}
& \psi=c v x^{3 / 4}(1+\xi)^{-1 / 4} f(\xi, \eta), \quad \eta=\frac{c y(1+\xi)^{-1 / 4}}{x^{1 / 4}} \\
& T-T_{\infty}=\left(T_{0}-T_{\infty}\right) \theta(\xi, \eta), \quad c=\left[\frac{g \beta\left(T_{0}-T_{\infty}\right)}{v^{2}}\right]^{1 / 4}
\end{aligned}
$$

and we get the following equations

$$
\begin{gathered}
(1+\varepsilon \theta) f^{\prime \prime \prime}-\varepsilon \theta^{\prime} f^{\prime \prime}+(1+\varepsilon \theta)^{2}\left\{\frac{3+2 \xi}{4(1+\xi)} f f^{\prime \prime}-\frac{1}{2(1+\xi)} f^{\prime 2}\right. \\
\left.+(1+\xi) \theta-\xi^{1 / 2}(1+\xi)^{1 / 2} f^{\prime}\right\} \\
=(1+\varepsilon \theta)^{2} \xi\left(f^{\prime} \frac{\partial f^{\prime}}{\partial \xi}-f^{\prime \prime} \frac{\partial f}{\partial \xi}\right) \\
\theta^{\prime \prime}+\operatorname{Pr} \frac{3+2 \xi}{4(1+\xi)} f \theta^{\prime}=\operatorname{Pr} \xi\left(f^{\prime} \frac{\partial \theta}{\partial \xi}-\theta^{\prime} \frac{\partial f}{\partial \xi}\right)
\end{gathered}
$$

with the no-slip boundary conditions

$$
\begin{aligned}
& f=f^{\prime}=0 ; \quad \theta=1 \quad \text { at } \eta=0 \\
& f^{\prime} \rightarrow 0 ; \quad \theta \rightarrow 0 \quad \text { as } \quad \eta \rightarrow \infty \\
& f=\theta=0 ; \quad \text { at } \quad \xi=0, \eta\rangle 0
\end{aligned}
$$

Here the coefficient of skin-friction, $\tau$, and the coefficient of the rate of heat transfer, $Q$ are defined as follows.

$$
\begin{aligned}
& \tau=\frac{H_{0}}{g \beta\left(T_{0}-T_{\infty}\right)}\left(\frac{\sigma v}{\rho_{\infty}}\right)^{1 / 2}\left(\frac{\partial u}{\partial y}\right)_{y=0}=\xi^{1 / 4}(1+\xi)^{-3 / 4} f^{\prime \prime}(\xi, 0) \\
& Q=\frac{1}{\left(T_{0}-T_{\infty}\right)}\left(\frac{\rho_{\infty} v}{\sigma H_{0}^{2}}\right)^{1 / 2}\left(\frac{\partial T}{\partial y}\right)_{y=0}=\xi^{-1 / 4}(1+\xi)^{-1 / 4} \theta^{\prime}(\xi, 0)
\end{aligned}
$$

\section{Solution Near the Leading Edge for Small $\xi$}

For small $\xi$ from the equations (7) and (8) we approximate the following equations

$$
\begin{gathered}
(1+\varepsilon \theta) f^{\prime \prime \prime}-\varepsilon \theta^{\prime} f^{\prime \prime}+(1+\varepsilon \theta)^{2}\left\{\frac{3}{4} f f^{\prime \prime}-\frac{1}{2} f^{\prime 2}+\theta-\xi^{1 / 2} f^{\prime}\right\} \\
=(1+\varepsilon \theta)^{2} \xi\left(f^{\prime} \frac{\partial f^{\prime}}{\partial \xi}-f^{\prime \prime} \frac{\partial f}{\partial \xi}\right)
\end{gathered}
$$




$$
\theta^{\prime \prime}+\frac{3}{4} \operatorname{Pr} f \theta^{\prime}=\operatorname{Pr} \xi\left(f^{\prime} \frac{\partial \theta}{\partial \xi}-\theta^{\prime} \frac{\partial f}{\partial \xi}\right)
$$

where the boundary conditions (9) remains the same.

To get the solutions of the above Eqs. (10) and (11) we use the $2^{\text {nd }}$ and $3^{\text {rd }}$ order series solution and the finite difference method.

\section{Series solution method}

For series solution we consider the following series

$$
\begin{aligned}
& f(\xi, \eta)=f_{0}+\xi^{1 / 2} f_{1}+\xi f_{2}+\cdots \cdots \\
& \theta(\xi, \eta)=\theta_{0}+\xi^{1 / 2} \theta_{1}+\xi \theta_{2}+\cdots \cdots
\end{aligned}
$$

where $f_{0}, \theta_{0}$ are the well known free convection similarity solutions for flow around a constant temperature semi-infinite vertical plate and where $f_{1}, \theta_{1}$ are effectively the first order correction and $f_{2}, \theta_{2}$ are effectively second order correction to the flow due to the presence of magnetic field.

Using (12) and (13) in (10) and (11) respectively we get the following equations:

For the coefficient of $\xi^{0}$

$$
\begin{gathered}
\left(1+\varepsilon \theta_{0}\right) f_{0}^{\prime \prime \prime}-\varepsilon \theta_{0}^{\prime} f^{\prime \prime}+\left(1+\varepsilon \theta_{0}\right)^{2}\left\{\frac{3}{4} f_{0} f_{0}^{\prime \prime}-\frac{1}{2} f_{0}^{\prime 2}+\theta_{0}\right\}=0 \\
\theta_{0}^{\prime \prime}+\frac{3}{4} \operatorname{Pr} f_{0} \theta_{0}^{\prime}=0
\end{gathered}
$$

with the boundary conditions

$$
\begin{aligned}
& f_{0}=f_{0}^{\prime}=0 ; \quad \theta_{0}=1 \quad \text { at } \eta=0 \\
& f_{0}^{\prime} \rightarrow 0 ; \quad \theta_{0} \rightarrow 0 \quad \text { as } \eta \rightarrow \infty \\
& f_{0}=\theta_{0}=0 ; \quad \text { at } \xi=0, \eta>0
\end{aligned}
$$

For the coefficients of $\xi^{1 / 2}$

$$
\begin{aligned}
& \left(1+\varepsilon \theta_{0}\right) f_{1}^{\prime \prime \prime}+\varepsilon\left(\theta_{1} f_{0}^{\prime \prime \prime}-\theta_{0}^{\prime} f_{1}^{\prime \prime}-\theta_{1}^{\prime} f_{0}^{\prime \prime}\right) \\
& +2 \varepsilon \theta_{1}\left(1+\varepsilon \theta_{0}\right)\left\{\frac{3}{4} f_{0} f_{0}^{\prime \prime}-\frac{1}{2} f_{0}^{\prime 2}+\theta_{0}\right\} \\
& +\left(1+\varepsilon \theta_{0}\right)^{2}\left\{\frac{3}{4} f_{0} f_{1}^{\prime \prime}+\frac{5}{4} f_{0}^{\prime \prime} f_{1}-\frac{3}{2} f_{0}^{\prime} f_{1}^{\prime}+\theta_{1}-f_{0}^{\prime}\right\}=0 \\
& \theta_{1}^{\prime \prime}+\operatorname{Pr}\left\{\frac{3}{4} f_{0} \theta_{1}^{\prime}+\frac{5}{4} f_{1} \theta_{0}^{\prime}-\frac{1}{2} f_{0}^{\prime} \theta_{1}\right\}=0
\end{aligned}
$$


with the boundary conditions

$$
\begin{aligned}
& f_{1}=f_{1}^{\prime}=0 \quad ; \theta_{1}=1 \quad \text { at } \eta=0 \\
& f_{1}^{\prime} \rightarrow 0 \quad ; \theta_{1} \rightarrow 0 \quad \text { as } \eta \rightarrow \infty \\
& \left.f_{1}=\theta_{1}=0 \quad ; \text { at } \xi=0, \eta\right\rangle 0
\end{aligned}
$$

For the coefficients of $\xi$

$$
\begin{aligned}
& \left(1+\varepsilon \theta_{0}\right) f_{2}^{\prime \prime \prime}+\varepsilon\left(\theta_{1} f_{1}^{\prime \prime \prime}+\theta_{2} f_{0}^{\prime \prime \prime}-\theta_{0}^{\prime} f_{2}^{\prime \prime}+\theta_{1}^{\prime} f_{1}^{\prime \prime}-\theta_{2}^{\prime} f_{0}^{\prime \prime}\right) \\
& +\left\{2 \varepsilon \theta_{2}\left(1+\varepsilon \theta_{0}\right)-\varepsilon^{2} \theta_{1}^{2}\right\}\left\{\frac{3}{4} f_{0} f_{0}^{\prime \prime}-\frac{1}{2} f_{0}^{\prime 2}+\theta_{0}\right\} \\
& +2 \varepsilon \theta_{1}\left(1+\varepsilon \theta_{0}\right)\left\{\frac{3}{4} f_{0} f_{1}^{\prime \prime}+\frac{5}{4} f_{0}^{\prime \prime} f_{1}-\frac{3}{2} f_{0}^{\prime} f_{1}^{\prime}+\theta_{1}-f_{0}^{\prime}\right\}+ \\
& \left(1+\varepsilon \theta_{0}\right)^{2}\left\{\frac{3}{4} f_{0} f_{2}^{\prime \prime}+\frac{5}{4} f_{1} f_{1}^{\prime \prime}+\frac{7}{4} f_{0}^{\prime \prime} f_{2}+\theta_{2}-f_{1}^{\prime}-f_{1}^{\prime 2}-2 f_{0}^{\prime} f_{2}^{\prime}\right\}=0 \\
& \theta_{2}^{\prime \prime}+\operatorname{Pr}\left(\frac{3}{4} f_{0} \theta_{2}^{\prime}+\frac{5}{4} f_{1} \theta_{1}^{\prime}-f_{0}^{\prime} \theta_{2}+\frac{7}{4} f_{2} \theta_{0}^{\prime}-\frac{1}{2} f_{1}^{\prime} \theta_{1}\right)=0
\end{aligned}
$$

with the boundary conditions

$$
\begin{aligned}
& f_{2}=f_{2}^{\prime}=0 ; \quad \theta_{2}=0 \quad \text { at } \eta=0 \\
& f_{2}^{\prime} \rightarrow 0 ; \quad \theta_{2} \rightarrow 0 \quad \text { as } \eta \rightarrow \infty \\
& \left.f_{2}=\theta_{2}=0 ; \quad \text { at } \xi=0, \eta\right\rangle 0
\end{aligned}
$$

Here skin friction and the rate of heat transfer are be of the following form

$$
\begin{gathered}
\tau=\frac{H_{0}}{g \beta\left(T_{0}-T_{\infty}\right)}\left(\frac{\sigma v}{\rho_{\infty}}\right)^{1 / 2}\left(\frac{\partial u}{\partial y}\right)_{y=0}=\xi^{1 / 4}\left(f_{0}{ }^{\prime \prime}+\xi^{1 / 2} f_{1}^{\prime \prime}+\cdots\right) \\
Q=\frac{1}{\left(T_{0}-T_{\infty}\right)}\left(\frac{\rho_{\infty} v}{\sigma H_{0}^{2}}\right)^{1 / 2}\left(\frac{\partial T}{\partial y}\right)_{y=0}=\xi^{-1 / 4}\left(\theta_{0}{ }^{\prime}+\xi^{1 / 2} \theta_{1}{ }^{\prime}+\cdots\right)
\end{gathered}
$$

\section{For Large $\xi$ Solution}

For large $\xi$ from the Eqs. (7) and (8) we can approximate the following equations

$$
\begin{gathered}
(1+\varepsilon \theta) f^{\prime \prime \prime}-\varepsilon \theta^{\prime} f^{\prime \prime}+(1+\varepsilon \theta)^{2}\left\{\frac{1}{2} f f^{\prime \prime}+\xi\left(\theta-f^{\prime}\right)\right\} \\
=(1+\varepsilon \theta)^{2} \xi\left(f^{\prime} \frac{\partial f^{\prime}}{\partial \xi}-f^{\prime \prime} \frac{\partial f}{\partial \xi}\right)
\end{gathered}
$$




$$
\theta^{\prime \prime}+\frac{1}{2} \operatorname{Pr} f \theta^{\prime}=\operatorname{Pr} \xi\left(f^{\prime} \frac{\partial \theta}{\partial \xi}-\theta^{\prime} \frac{\partial f}{\partial \xi}\right)
$$

with the boundary conditions

$$
\begin{aligned}
& f=f^{\prime}=0 ; \quad \theta=1 \quad \text { at } \eta=0 \\
& f^{\prime} \rightarrow 0 ; \quad \theta \rightarrow 0 \quad \text { as } \eta \rightarrow \infty \\
& f=\theta=0 ; \quad \text { at } \xi=0, \eta>0
\end{aligned}
$$

For convenient here we can write the expression for $\tau$ and $\mathrm{Q}$ as given bellow

$$
\begin{aligned}
& \tau=\xi^{-1 / 2} f^{\prime \prime}(\xi, 0) \\
& Q=\xi^{-1 / 2} \theta^{\prime}(\xi, 0)
\end{aligned}
$$

Here the skin friction and the rate of heat transfer for all the three cases are given in Table 1 and Table 2, respectively.

\section{Result and Discussion}

In this section we discuss the results obtained from the solution of the equations governing the MHD free convection flow of a viscous incompressible and electrically conducting fluid with temperature depend viscosity and constant thermal conductivity in the presence of uniformly distributed transverse magnetic field along the impermeable vertical flat plate. The solutions of the governing non-similar equations are obtained by three distinct methods, the extensive series solution method for small $\xi$, the implicit finite difference method or simply Keller box method for all $\xi$ and asymptotic solution method for large $\xi$.

Table 1. Numerical values of skin friction coefficient obtained by different methods for different $\varepsilon$ with with $\operatorname{Pr}=0.05$.

\begin{tabular}{lcccccc}
\hline$\xi$ & \multicolumn{3}{c}{$\varepsilon=0.5$} & & \multicolumn{3}{c}{$\varepsilon .0$} \\
\cline { 2 - 6 } & series & finite & asymptotic & series & finite & asymptotic \\
\hline 0.10 & 0.7898 & 0.8187 & & 0.9354 & 0.9342 & \\
0.20 & 0.8987 & 0.9881 & & 1.0361 & 1.0341 & \\
0.30 & 0.9482 & 0.9697 & & 1.1208 & 1.1189 & \\
0.40 & 0.9872 & 0.9994 & & 1.1543 & 1.1568 & \\
0.50 & 1.0162 & 1.0178 & & 1.1999 & 1.1887 & \\
0.60 & 1.0392 & 1.0294 & & 1.2265 & 1.2178 & \\
0.70 & 1.0582 & 1.0365 & & 1.2483 & 1.2399 & \\
0.80 & 1.0743 & 1.0407 & & 1.2669 & 1.2544 & \\
0.90 & 1.0885 & 1.0428 & & 1.2832 & 1.2699 & \\
1.00 & 1.1011 & 1.0443 & & 1.2978 & 1.2747 & \\
3.00 & & 0.9943 & 1.0610 & & 1.6247 & 1.6523 \\
4.00 & & 0.9707 & 1.0199 & & 1.7504 & 1.8060 \\
5.00 & & 0.9534 & 0.9896 & & 1.9801 & 1.9820 \\
6.0 & & 0.9399 & 0.9672 & & 2.1758 & 2.1790 \\
7.0 & & 0.9294 & 0.9511 & & 2.2942 & 2.2950 \\
8.0 & & 0.9210 & 0.9385 & & 2.3599 & 2.3604 \\
9.0 & & 0.9142 & 0.9289 & & 2.5459 & 2.5462 \\
10.0 & 0.9206 & 0.9207 & & 2.8824 & 2.8823 \\
\hline
\end{tabular}


The solutions of the present problem with wide range of the pertinent parameter $\xi$ are obtained by the finite difference method. The effect of the viscosity variation parameter $\varepsilon$ on the local skin friction and the local rate of heat transfer coefficient $\mathrm{Q}$ against the magnetic field parameter $\xi$ for the low prandtl number fluid with $\operatorname{Pr}=0.05$ (for lithium) are depicted in the Table 1 and in the Table 2 respectively. This problem is appropriate not only for lithium but also for any other fluid of very low prandtl number.

Table 2. Numerical values rate of heat transfer coefficient obtained by different methods for different $\varepsilon$ with with $\operatorname{Pr}=0.05$.

\begin{tabular}{lcccccc}
\hline$\xi$ & \multicolumn{3}{c}{$\varepsilon=0.5$} & & & $\varepsilon=1.0$ \\
& series & finite & asymptotic & series & finite & asymptotic \\
\hline 0.10 & 0.2125 & 0.2119 & & 0.2052 & 0.2025 & \\
0.20 & 0.1736 & 0.1713 & & 0.1762 & 0.1642 & \\
0.30 & 0.1534 & 0.1602 & & 0.1559 & 0.1442 & \\
0.40 & 0.1410 & 0.1406 & & 0.1426 & 0.1396 & \\
0.50 & 0.1303 & 0.1355 & & 0.1328 & 0.1211 & \\
0.60 & 0.1226 & 0.1272 & & 0.1251 & 0.1134 & \\
0.70 & 0.1164 & 0.1204 & & 0.1188 & 0.1071 & \\
0.80 & 0.1111 & 0.1147 & & 0.1135 & 0.1018 & \\
0.90 & 0.1065 & 0.1097 & & 0.1090 & 0.0972 & \\
1.00 & 0.1026 & 0.1054 & & 0.1050 & 0.0931 & \\
3.00 & & 0.0660 & 0.0724 & & 0.0533 & 0.0544 \\
4.00 & & 0.0575 & 0.0617 & & 0.0452 & 0.0458 \\
5.00 & & 0.0516 & 0.0544 & & 0.0388 & 0.0392 \\
6.0 & & 0.0471 & 0.0487 & & 0.0333 & 0.0341 \\
7.0 & & 0.0435 & 0.0451 & & 0.0285 & 0.0294 \\
8.0 & & 0.0407 & 0.0419 & & 0.0252 & 0.0261 \\
9.0 & & 0.0383 & 0.0391 & & 0.0225 & 0.0233 \\
10.0 & & 0.0363 & 0.0371 & & 0.0206 & 0.0207 \\
\hline
\end{tabular}

From Table 1 it can be seen that the skin friction increase due to the increase of viscosity variation parameter $\varepsilon$ and this increase is sufficiently large in the downstream regime that the regime far away from the leading edge. It is clear from Table 2 that the rate of heat transfer decrease due to increasing values of the viscosity variation parameter. In Tables 1 and 2, we see that the series solution converge to the finite difference method solution for small values of $\xi$ and the asymptotic solutions converge with the solutions of finite difference for large $\xi$. As for example if $\xi=0.2$, the result from the series solution and finite difference are much closed and for $\xi=10$, the result from the asymptotic solution and finite difference are much closed. Again the increasing values of the magnetic field parameter $\xi$ the values of both the skin friction and the rate of heat transfer decrease. 
The profiles for velocity and viscosity defined in Eqs. (26) and (27) for velocity and viscosity, respectively are shown in Fig. 2 and Fig. 3 for different values of $\xi$ and $\varepsilon$.

$$
\begin{aligned}
& u G r_{x}^{1 / 4} H_{v, x}^{-1}=\xi^{1 / 2}(1+\xi)^{-1 / 2} f^{\prime}(\eta, \xi) \\
& \text { where } G r_{x}=\frac{g \beta\left(T_{0}-T_{\infty}\right) x^{3}}{v^{2}}, H_{v, x}=\left(\frac{\sigma H_{0}^{2}}{\rho_{\infty}}\right)^{2} x \\
& \frac{\mu}{\mu_{0}}=\frac{1}{1+\varepsilon \theta}
\end{aligned}
$$

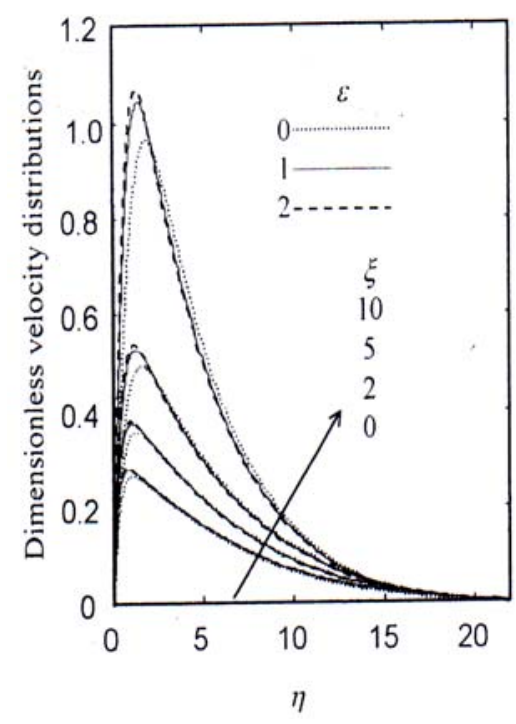

Fig. 2. Velocity profiles for different values of $\xi$ and $\varepsilon$ against $\eta$.

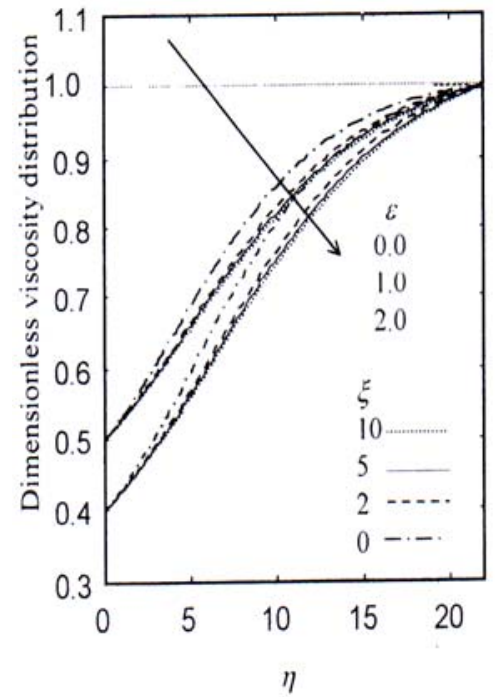

Fig. 3. Viscosity profiles for different values of $\xi$ and $\varepsilon$ against $\eta$.

From Fig. 2 it can be seen that the velocity profiles increase due to the increase of viscosity variation parameter, $\varepsilon$ and it smoothly decreases at the downstream regime. Further one can see that the velocity profiles decrease due to increase of magnetic field parameter $\xi$. We observe that there exists a maximum for each of $\varepsilon$, i.e. for $\varepsilon=0.0,1.0$ and 2.0. The corresponding local maxima occur at $\eta \sim 1.583,1.237$ and 1.144 with $\xi=$ 2.0. Similarly we see that there exist different local maxima for different graphs of the velocity profiles for all other values of $\xi=0,5$ and 10 with $\varepsilon=0.0,1.0$ and 2.0 respectively against $\eta$ near the surface of the vertical plate. Fig. 3 shows that the profiles for viscosity decrease with increasing values of both the viscosity variation parameter $\varepsilon$ and the magnetic field parameter $\xi$. But the effect is not significant at the surface of the plate. All these profiles tend to a limiting value on the profile for $\varepsilon=0.0$ which is unity. 


\section{References}

1. E. M. Sparrow and J. L. Gregg, ASME J. Appl. Mech. 83, 133 (1959).

2. J. H. Merkin, J. Fluid Mech. 35, 439 (1969). doi:10.1017/S0022112069001212

3. J. R. Lloyd and E. M. Sparrow, Int. J. Heat Mass Transfer 13, 434 (1970).

4. G. Wilks and R. Hunt, J. Comp. Phys. 40, (1981)

5. M. S. Raju, X. Q. Liu and C. K. Law, Int. J. Heat Mass Transfer 27, 2215 (1984). doi:10.1016/0017-9310(84)90080-2

6. G. Tingwei, R. Bachrun and M. Daguenet, Int. J. Heat Mass Transfer 25, 1061 (1982). doi:10.1016/0017-9310(82)90081-3

7. E. M. Sparrow and R. D. Cess, Int. J. Heat Mass Transfer 3, 267 (1961). doi:10.1016/0017-9310(61)90042-4

8. N. Riley, J. Fluid Mech. 18, 267 (1964). doi:10.1017/S0022112064000416

9. H. K. Kuiken, J. Fluid Mech. 40, 21 (1970). doi:10.1017/S0022112070000022

10. K. R. Sing and T. G. Cowling, J. Mech. Appl. Math. 16, 1 (1963). doi:10.1093/qjmam/16.1.1

11. E. M. Crammer and S. I. Pai, Megneto fluid Dynamics for Engineering and Applied Physicists (McGraw-Hill, New York, 1974).

12. N. P. Sing, A. K. Sing and A. K. Sing, The Arabian Journal for Science and Engineering 32, 1A, 93 (2007).

13. M. A. Hossain and M. Ahmed, Int. J. Heat Mass Transfer 33, 571 (1990). doi:10.1016/0017-9310(90)90190-6

14. R. N. Rat and S. Chaudhary, Applied Mathematical Sciences 26 (3), 1285 (2009).

15. M. A. Samad and M. Mohebujjaman, Research Journal of Applied Sciences, Engineering and Technology 1 (3), 98 (2009).

16. R. Taneja and N. C. Jain, Defense Science Journal 54 (1), 21 (2004).

17.. M. A. Hossain, I. Pop and M. Ahmed, J. Theo. Appl. Fluid Mech. 1, 194 (1996).

18. T. Cebeci and P. Bradshaw, Physical and computational aspects of convective heat transfer (Springer, New York, 1984).

19. G. Wilks, J. Appl. Math. Phys., 27, 621 (1976). doi:10.1007/BF01591174

20. M. A. El-Hakiem and M. M. M. Abdou, International Journal of Applied Mechanics and Engineering 11(2), 301 (2006).

21. R. Kandasamy, I. M. Hashim and Seripah, Theoretical Applied Mechanics, Belgrade 34 (2), 111 (2007). doi:10.2298/TAM0702111K

22. S. F. Ahmmed and M. S. Alam Sarker, Journal of Mechanics of Continua and Mathematical Sciences, 4 (2), 530 (2010).

23. J. Gray,D. R. Kassory, H. Tadjeran and A. Zebib, J. Fluid Mech. 117, 233 (1982). doi:10.1017/S0022112082001608

24. K. N. Mehta and S. Sood, Int. J. Engn. Scie. 30, 1083 (1992). doi:10.1016/0020-7225(92)90032-C

25. N. G. Kafoussias and E. W. Williams, Acta Mechanica 110, 123 (1997). doi:10.1007/BF01215420

26. N. G. Kafoussias, D. A. S. Rees and J. E. Daskalakis, Acta Mechanica 127, 39 (1998). doi:10.1007/BF01170361 